$(=\rho(x+L))$ on $0 \leqq x<L$ by:

$$
\rho(x)= \begin{cases}1, & 0 \leqq x<d, \\ k, & (2 k-3) d \leqq x<(2 k-1) d \quad(k=2, \cdots, n), \\ b, & (2 n-1) d \leqq x<2 n d,\end{cases}
$$

where $b$ is a positive constant to be determined. Then the graph of $P(x)$ is a polygonal path with vertices having abscissas on $0 \leqq x<L$ at $x=0, d, \cdots,(2 n-1) d$. It will be shown that for a proper choice of the constant $b$ the function $P(x)$ of (1) has maxima occurring at the points $x=0,2 d, \cdots,(2 n-2) d$. For reasons of continuity, it is clear that the above $\rho(x)$ can be replaced by a continuous positive function possessing a single maximum on the interval $0 \leqq x \leqq L$ and so that the corresponding $P(x)$ of (1) possesses $n$ maxima on $0 \leqq x<L$; see the remark of [1], p. 272 .

An easy calculation shows that, for the $\rho(x)$ of $(2)$, the function $P(x)$ satisfies

$$
P(m d)=c_{m}+\left\{\begin{array}{lll}
(n+1) b d, & \text { for } & m=1,3, \cdots, 2 n-1, \\
(n+2) b d, & \text { for } & m=2,4, \cdots, 2 n-2 .
\end{array}\right.
$$

Accordingly, since the constants $c_{m}$ do not depend on $b$, it is clear from (3) that for a sufficiently large $b$, the inequalities $P(2 k d)>P[(2 k+1) d]$ and $P[(2 k+1) d]<P[(2 k+$ $2) d]$ hold for $k=0,1,2, \cdots, n-1$. Thus, the function $P(x)$ has $n$ maxima on $0 \leqq x<L$ as claimed, and the existence of a continuous $\rho(x)$ with the desired properties now follows as remarked above.

\title{
REFERENCES
}

[1] P. Hartman and A. Wintner, On the maxima of the Patterson functions, Phys. Rev. 81, 271-273 (1951). [2] A. L. Patterson, A direct method for the determination of the components of interatomic distances in crystals, Z. Krist. 90, 517-542 (1935).

\section{ON HELICAL SPRINGS OF FINITE THICKNESS*}

\section{BY PETER HENRICI (National Bureau of Standards)}

1. Introduction. The problem of determining the stress distribution in a statically loaded, closely coiled helical spring, whose cross-section is not necessarily small in comparison with the diameter of the helix, has been solved approximately by iteration by Göhner $[5,6]$ and exactly in terms of a series of appropriate Legendre functions by Freiberger [4]. In the present paper it is shown that the exact values of the stress concentration factor as well as of some other significant quantities of the problem can be developed in terms of a certain parameter (namely the reciprocal of the so-called spring index) so as to recover Göhner's approximations and to obtain further approximations of the same type.

*Received May 20, 1954. The preparation of this paper was sponsored by the Office of Naval Research. 
2. Formulation of the problem. Mathematically the stress problem under consideration may be stated as follows ${ }^{1}$. Let the $z$-axis of a system of cylindrical coordinates $(r, \vartheta, z)$ coincide with the spring axis and denote by $\mathcal{G}$ the intersection of a turn of the spring with the half-plane $\vartheta=0$. Let the center of gravity of $\varrho$ lie on the plane $z=0$ at a distance $R$ from the $z$-axis. Let furthermore

$$
C=\frac{G \sin 2 \alpha}{R},
$$

where $G$ is the shear modulus of elasticity and $\alpha$ the difference of the pitch angles of the spring in loaded and unloaded state ${ }^{2}$. Then, if the "stress function" $\phi(r, z)$ solves the boundary value problem

$$
\begin{gathered}
\frac{\partial^{2} \phi}{\partial r^{2}}+\frac{\partial^{2} \phi}{\partial z^{2}}-\frac{3}{r} \cdot \frac{\partial \phi}{\partial r}=1 \text { in } \mathcal{G}, \\
\phi=0 \quad \text { on the boundary of } \mathcal{G},
\end{gathered}
$$

the shear stresses $\left(\tau_{r}, \tau_{z}\right)$ in $\mathcal{G}$ are given by

$$
\tau_{r}=-C \frac{R^{2}}{r^{2}} \frac{\partial \phi}{\partial z}, \quad \tau_{z}=C \frac{R^{2}}{r^{2}} \frac{\partial \phi}{\partial r} .
$$

Here $C$, because it involves the unknown quantity $\alpha$, is unknown. If one wishes to compute the maximum shear stress occurring under a given load,

$$
\tau_{\max }=C \tau_{\max }^{*}, \quad \tau_{\max }^{*}=\operatorname{Max}(R / r)^{2}\left[\left(\frac{\partial \phi}{\partial r}\right)^{2}+\left(\frac{\partial \phi}{\partial z}\right)^{2}\right]^{1 / 2},
$$

one has to eliminate $C$ from (3) and from the expression for the total moment, ${ }^{3}$

$$
\begin{gathered}
W=C W^{*}, \\
W^{*}=R^{2} \iint_{\delta} r^{-2}\left[(R-r) \frac{\partial \phi}{\partial r}-z \frac{\partial \phi}{\partial z}\right] d r d z=-2 R^{3} \iint_{\S} r^{-3} \phi d r d z,
\end{gathered}
$$

for which by mechanical considerations one can also write

$$
W=R F,
$$

where $F$ denotes the force exerted on the spring in the direction of the $z$-axis. It follows that

$$
\tau_{\max }=R F\left|\frac{\tau_{\max }^{*}}{W^{*}}\right| \text {. }
$$

3. The stress-concentration factor. We are here primarily interested in the case where $\mathcal{G}$ is a circle. If we denote its radius by $\rho$, it is convenient to write

$$
\tau_{\max }=K_{c} \tau_{\max }^{(0)} \text {, }
$$

${ }^{1}$ Mitchell [8]; Göhner [5, 6]; Biezeno and Grammel [2], pp. 306-328; Southwell [9], p. 374; Timoshenko and Goodier [10], pp. 391-395; Wahl [11], pp. 38-42; see also Collatz [3], pp. 292, 310, 313, 355, 363, where the problem is used as an example for the application of various numerical methods.

'The theory is exact only if the pitch angle of the spring is zero, i.e., if the spring reduces to an incomplete tore. However, as the quoted authors point out, it may be regarded as a good approximation in the case of a small pitch angle. For a detailed discussion of this point see Wahl [11], p. 41.

${ }^{3}$ Mitchell [8], p. 134. 
where

$$
\tau_{\max }^{(0)}=\frac{2 R F}{\pi \rho^{3}}
$$

is the maximum shear stress as given by the well-known elementary theory (neglecting the finite thickness or, what amounts to the same, the curvature of the spring), and $K_{c}$ is the so-called stress-concentration factor ${ }^{4}$. Since in this case it is clear for mechanical reasons that $\tau_{\max }$ occurs at the point $P$ of the circumference of the circle nearest to the spring axis, we obtain from (5), (6) and (7), denoting the shear stress at this point by $\tau_{P}=C \tau_{P}^{*}$,

$$
K_{c}=\frac{\pi \rho^{3}}{2}\left|\frac{\tau_{P}^{*}}{W^{*}}\right| .
$$

4. Solution of the boundary value problem for a circle. As indicated by Mitchell [8], an explicit solution of the boundary value problem (1) can be given for the case that $\mathcal{G}$ is a circle if bipolar coordinates $(\xi, \eta)$ defined by

$$
r=\frac{c \sinh \xi}{\cosh \xi-\cos \eta}, \quad z=\frac{s \sinh \eta}{\cosh \xi-\cos \eta}
$$

( $c=$ const.) are introduced. It is easily seen that if $\xi_{0}>0$ is a fixed value of $\xi$ and $\eta$ varies from 0 to $2 \pi$, the point $(r, z)$ travels along a given circle with center at $(0, R)$ and radius $\rho<R$ provided

$$
\cosh \xi_{0}=R / \rho, \quad c=\left(R^{2}-\rho^{2}\right)^{1 / 2} .
$$

For the following it is convenient to denote by

$$
q=\rho / R
$$

the reciprocal value of the so-called spring index. The solution of (1), which in essence was first given explicitly by Freiberger [4] (who, however, uses a stress function slightly different from $\phi)$, can then be written as follows:

$$
\begin{aligned}
\phi(r, z)=2^{-3 / 2} \pi^{-1}\left(R^{2}-\rho^{2}\right) & (\cosh \xi-\cos \eta)^{-3 / 2} \sinh ^{2} \xi \\
& \cdot \sum_{n=0}^{\infty} \epsilon_{n}\left\{A_{n}(q) Q_{n-1 / 2}^{2}(\cosh \xi)-Q_{n-1 / 2}(\cosh \xi)\right\} \cos n \eta .
\end{aligned}
$$

Here $\epsilon_{n}$ is the Neumann number $\left(\epsilon_{0}=1 ; \epsilon_{n}=2, n=1,2,3, \cdots\right)$,

$$
A_{n}(q)=Q_{n-1 / 2}\left(q^{-1}\right) / Q_{n-1 / 2}^{2}\left(q^{-1}\right),
$$

and $Q_{v}(z)$ and $Q_{v}^{\mu}(z)$ denote the ordinary and the associated Legendre function of the second kind as defined in [7]. It can be shown that the series in (10) converges uniformly in $\xi$ and $\eta$ for all $\eta$ and all $\xi$ such that $\cosh ^{-1} \xi \leqq q<1$.

From (2) and (10) one readily derives the following expansion for $\tau_{P}^{*}$, which is equivalent to that given in [4]:

$$
\begin{aligned}
\tau_{P}^{*}=2^{-3 / 2} \pi^{-1} \rho q^{-3 / 2}(1-q)^{-1}(1+q)^{1 / 2} \\
\cdot \sum_{n=0}^{\infty}(-1)^{n} \epsilon_{n}\left\{(n-3 / 2) A_{n}(q) Q_{n+1 / 2}^{2}\left(q^{-1}\right)-(n+1 / 2) Q_{n+1 / 2}\left(q^{-1}\right)\right\} .
\end{aligned}
$$

${ }^{4}$ Wahl [11], p. 110. 
Similarly one finds from (4) and (10)

$$
W^{*}=W^{*(0)}+W^{*(1)},
$$

where

$$
W^{*(1)}=\pi \rho^{4} q^{-4}\left[1-\left(1-q^{2}\right)^{1 / 2}\right],
$$

whilst $W^{*(0)}$ is given by the more complicated expression

$$
\begin{gathered}
W^{*(0)}=-2 \pi^{-1} \rho^{4} q^{-4}\left(1-q^{2}\right)^{1 / 2} \sum_{n=0}^{\infty} \epsilon_{n} A_{n}(q) S_{n}(q), \\
S_{n}(q)=\int_{1 / q}^{\infty}\left(s^{2}-1\right)^{-1} Q_{n-1 / 2}(s) Q_{n-1 / 2}^{2}(s) d s .
\end{gathered}
$$

These formulas can again be shown to be equivalent to those given by Freiberger. Formula (15) has the numerical advantage over the corresponding one in [4] of not involving differences of large numbers ${ }^{5}$.

5. Developments in powers of $q$. In order to bring the above results into a practical form, we develop the right sides of (12) and (13) in terms of powers of the only essential parameter $q$. This is done by developing the Legendre series termwise and rearranging. 'The rearrangement, which is permissible in view of Weierstrass' theorem, is facilitated by the fact that the $n$th terms in (12) and (15) are $0\left(q^{n}\right)$ and $0\left(q^{2 n+2}\right)$ respectively, so that in collecting powers of a fixed order one has to cope only with a finite number of terms. Computational details are omitted.

We start by developing $A_{n}(q)$. Using (11) we obtain by straightforward division of the two hypergeometric series involved in the definition of the Legendre functions

$$
\begin{aligned}
A_{n}(q)=\frac{1}{(n+1 / 2)(n+3 / 2)}\left\{1-\frac{1}{n+1} q^{2}-\frac{12 n^{2}+8 n-3}{16(n+1)^{2}(n+2)} q^{4}\right. \\
\\
\left.-\frac{80 n^{4}+288 n^{3}+8 n^{2}-392 n+33}{128(n+1)^{3}(n+2)(n+3)} q^{6}-\cdots\right\} .
\end{aligned}
$$

It is now easily found that

$$
\tau_{P}^{*}=\frac{\rho}{2}\left\{1+\frac{5}{4} q+\frac{17}{16} q^{2}+\frac{215}{256} q^{3}+\frac{6827}{8192} q^{4}+\cdots\right\} .
$$

For $W^{*(1)}$ the coefficients of the power series can be given in closed form:

$$
W^{*(1)}=\frac{\pi \rho^{4}}{2 q^{2}} \sum_{n=0}^{\infty} \frac{(1 / 2)_{n}}{(n+1) !} q^{2 n} .^{6}
$$

In order to expand $W^{*(0)}$, we need to develop the integrals $S_{n}(q)$. By partial integration one finds

$$
S_{n}(q)=-\left.\frac{1}{2} \frac{d}{d s}\left[Q_{n-1 / 2}(s)\right]^{2}\right|_{--1 / q}-\int_{1 / q}^{\infty}\left(s^{2}-1\right)^{-1}\left[Q_{n-1 / 2}^{1}(s)\right]^{2} d s .
$$

sSee the remark in [4], p. 370.

${ }^{\circ}(a)_{0}=1 ;(a)_{n}=a(a+1) \ldots(a+n-1), n=1,2, \ldots$ 
The terms of this expression are easily expanded by use of the formula

$$
\begin{aligned}
& s^{2 \nu+2 \mu+2}\left(s^{2}-1\right)^{-\mu}\left[Q_{\nu}^{\mu}(s)\right]^{2} \\
& =e^{2 i \mu \pi}\left\{\frac{\Gamma(\nu+\mu+1) \Gamma(1 / 2)}{2^{\nu+1} \Gamma(\nu+3 / 2)}\right\}^{2} \sum_{n=0}^{\infty} \frac{(v+\mu+1)_{n}}{n !} s^{-2 n}{ }_{3} F_{2}\left[\begin{array}{c}
\nu-\mu+1, \nu+1,-n ; \\
2 \nu+2, \nu+3 / 2
\end{array}\right],
\end{aligned}
$$

which can be proved by application of a result due to Clausen ${ }^{7}$. Carrying out the expansions and rearranging, we finally find

$$
W^{*(0)}=-\frac{\pi \rho^{4}}{2 q^{2}}\left\{1-\frac{1}{4} q^{2}+\frac{1}{32} q^{4}-\frac{349}{24576} q^{6}+\cdots\right\}
$$

and hence, using (13),

$$
W^{*}=-\frac{\pi \rho^{4}}{4}\left\{1+\frac{3}{16} q^{2}+\frac{2269}{12288} q^{4}+\cdots\right\} .
$$

In virtue of (8) we obtain now for the stress-concentration factor the expression

$$
K_{c}=1+\frac{5}{4} q+\frac{7}{8} q^{2}+\frac{155}{256} q^{3}+\frac{11911}{24576} q^{4}+\cdots .
$$

6. Comparison with other approximate results. The first three terms of the last formula, as well as the corresponding terms in (18) and (22), agree with Göhner's second order approximation $[5,6]$, which was constructed by carrying out the first two steps of an iterative method of solving (1). See also [2] and [10] for expositions of the method. The third order term in (23) however does not agree with a term $+q^{3}$ given by Wahl. The error seems to be due to the fact that, following [10], Wahl writes (in our notation)

$$
\tau_{\max }^{*}=\frac{\rho}{2}\left\{\frac{1}{1-q}+\frac{1}{4} q+\frac{1}{16} q^{2}\right\},
$$

(which up to the term of second order in $q$ is identical with (18)) and then extends the expansion only of $(1-q)^{-1}$ to the third order term.

\section{BIBLIOGRAPHY}

1. W. N. Bailey, Generalized hypergeometric series, Cambridge, 1935.

2. C. B. Biezeno and R. Grammel, Technische Dynamik, Berlin, 1939.

3. L. Collatz, Numerische Behandlung von Differentialgleichungen, Springer, 1951.

4. W. Freiberger, The uniform torsion of an incomplete tore, Aust. J. Scient. Research A2, 354-375 (1949).

5. O. Göhner, Schubspannungsverteilung im Querschnitt einer Schraubenfeder, Ing. Arch. 1, 619-644 (1930).

6. O. Göhner, Schubspannungsverteilung im Querschnitt eines gedrillten Ringstabes, mit Anwendungen auf Schraubenfedern, Ing. Arch. 2, 1-19 (1931).

7. W. Magnus, und F. Oberhettinger, Formeln und Sätze für die speziellen Funktionen der mathematischen Physik, 2nd ed., Berlin, 1948.

8. J. H. Michell, The uniform torsion and flexure of incomplete tores, with applications to helical springs, Proc. Lond. Math. Soc. 31, 130-146 (1899).

9. R. V. Southwell, Some practically important stress systems in solids of revolution, Proc. Roy. Soc. A180, 367-396 (1942).

10. S. Timoshenko and J. N. Goodier, Theory of elasticity, 2nd. ed., New York, 1951.

11. A. M. Wahl, Mechanical springs, Cleveland, 1944.

'See Bailey [1], p. 86. We also adopt Bailey's notation for the generalized hypergeometric series.

${ }^{8} \mathrm{Wahl}[11]$, p. 42, formula (37). 